\title{
Perceptions and Attitudes of Midwives on Respectful Maternity Care During Childbirth: A Qualitative Study in Three District Hospitals of Kigali City of Rwanda, 2019
}

Valentine Uwamahoro ( $\square$ valmahoro@gmail.com )

IntraHealth: IntraHealth International Inc

Jean Paul Semasaka Sengoma

Umea Universitet

Albert Ndagijimana

University of Rwanda School of Public Health

James Humuza

University of Rwanda School of Public Health

Research

Keywords: Perceptions, attitudes, midwives, respectful maternity care, childbirth, Rwanda

Posted Date: August 6th, 2021

DOl: https://doi.org/10.21203/rs.3.rs-769349/v1

License: (c) (1) This work is licensed under a Creative Commons Attribution 4.0 International License.

Read Full License 


\section{Abstract}

Background: Respectful maternity care (RMC) is "a universal human right for every childbearing woman". Mistreatment during childbirth is a violation of women's fundamental rights and affects accessibility to women's health services. In Rwanda, little is known about health care professionals' perceptions and attitudes towards RMC during childbirth. This study aimed to investigate the perceptions and attitudes of midwives towards the provision of respectful maternity care during childbirth.

Methods: This qualitative study used individual in-depth interviews in Kinyarwanda language. A purposive sampling method was used to reach out to 28 midwives from three district hospitals in Kigali city. Interviews were recorded, transcribed, translated into English and thematic content analysis was performed using Atlas ti, version 7. The study was ethically approved by the University of Rwanda College of Medicine and Health Sciences Institutional Review Board, before data collection.

Results: Most of participants revealed that they have knowledge on RMC and perceive that they provide maternal health care based on women's rights. Positive attitudes towards providing RMC were reported by midwives working in maternity. However, a considerable number of participants reported the existence of abusive practices among midwives. Most of the midwives reported facing many challenges which affect their ability to provide respectful maternal care.

Conclusion: In general, midwives understand the seven rights of mothers and had a positive attitude towards providing RMC. However, some abusive practices still exist among midwives while providing RMC with considerable reported challenges, including overload and lack of labour monitoring materials. The adjustment of the ratio of midwives to clients and availability of essential materials in labour monitoring is suggested.

\section{Plain English Summary}

Respectful maternity care during childbirth is still a global concern especially in low income countries. Factors leading to disrespectful childbirth have already been studied amongst women yet there is a purcity of research done to study the perception of midwives towards respectful maternity care in different health settings. This study aimed to provide an overview on how midwives perceive respectful maternity care and their attitude in supporting mothers' during childbirth. One-on-one, fact-to-face interviews were conducted with midwives within three District hospitals of Kigali city in Rwanda. This study has added to the scope of knowledge on maternal health practices during childbirth. Findings were that midwives in the study setting are knowledgeable on respectful maternity care but faces some challenges that make them unable fully demonstrate these skills. These factors included work overload and lack of some essential equipment such as cardiotocography machine. Despite midwives skills on childbirth services, they are still some that render abusive services including shouting at mothers, ignoring their pain during labor or/and slapping them during the delivery process. This brings us to the conclusion that respectful maternity care during childbirth is not yet at an optimal level and stakeholders need to 
increase the number of midwives in respect to the desired midwives/clients ration to provide quality childbirth care. More so, efforts should be made to make available essential labor monitoring equipment and then improving maternity care service being delivered.

\section{Background}

Pregnancy and childbirth are two periods which place women at a substantial risk of mortality and morbidity, specifically in low-and middle-income countries (LMICs) [1]. Out of 303,000 maternal deaths that occurred worldwide in 2015, 99\% were from LMICs [2]. The reduction in maternal and new born mortality and morbidity is a global priority in Sustainable development goals (SDGs) [3].

The number of health facilities with childbirth services as a measure to reduce maternal mortality remains insufficient $[4,5]$. Therefore, the consideration of physical health and overall well-being of pregnant women during childbirth becomes imperative to ensure a positive impact on maternal health outcomes [6]. Traumatic birth experience contributes to profound consequences on maternal well-being [7].

The concept of "respectful maternity care (RMC)" acknowledges that women's experiences of childbirth are vital components of health healthcare quality [8]. According to the stated universal rights of childbearing women charter, every woman has the right to dignified, respectful sexual and reproductive healthcare, including during childbirth [9].

Mistreatment during childbirth can represent a violation of women's fundamental rights and serves as a powerful disincentive for women to seek healthcare in facilities for their subsequent deliveries and cannot be understood unless deep exploration is done on women's and health care providers' attitudes [1].

Therefore, helping mothers to have a positive childbirth experience is important for the woman's wellbeing and facilitates the mother-child bonding [10]. There is a need to address this issue by providing RMC with positive impact on women's labour and childbirth experience [11].

In Rwanda, few studies conducted in regard to RMC during childbirth assessed the perceptions of women in post-partum period on RMC offered by midwives through their childbirth experience $[10,12]$.

The only direct observational study conducted in of health facilities in East and Southern Africa, including Rwanda, to determine the prevalence of RMC found many women who had experienced poor interactions with providers and were not well-informed about their care, with both physical and verbal, with abandonment and neglect as the most frequent form of disrespect and abuse in the open-ended comments [8].

Little is known about healthcare professionals' perceptions and attitudes towards RMC during childbirth in Rwanda. This study complemented the information provided by existing studies conducted in Rwanda by providing documenting midwives' perceptions and attitudes during childbirth and showed the area for improvement in maternal and child healthcare. 


\section{Methods}

\section{Aims}

This study aimed to investigate the perceptions and attitudes of midwives towards the provision of RMC during childbirth. Specifically, it sought to investigate midwives' knowledge on the rights of women during childbirth, to identify midwives' attitudes towards respectful maternal care during childbirth and, to investigate midwives' knowledge on different kinds of mistreatments experienced by women during childbirth period.

\section{Design}

This was a qualitative cross sectional study, with an interview guide based on seven rights of women during childbirth, to gather the information about the perceptions and attitudes of midwives towards RMC during childbirth.

\section{Settings}

This study was conducted in three district hospitals from Kigali city: Masaka district hospital of Kicukiro District, Kacyiru district hospital of Gasabo district and Muhima district hospital of Nyarugenge district. The study area was selected due to a good number of deliveries that take place in Kigali city, with many midwives; thus expecting to gather various views among midwives who assist those deliveries. In a district where there are two district hospitals, one of them was randomly chosen. Data collection was conducted from1st June to 15th September 2019.

Sampling and study participants

A purposive sampling method was used. All midwives meeting the inclusion criteria in each selected district hospital were included in our study till we reached theoretical saturation. At the end of our data collection, we found that our sample size was between eight to ten midwives. Working in labour or delivery ward and being able to communicate in Kinyarwanda or English were inclusion criteria. Were excluded midwives on sick or annual leave and those who were absent during the time of data collection.

\section{Data collection}

We trained six data collectors; two per district (one moderator and one note taker).An interview guide including questions about the perceptions, attitudes and practices of midwives during birth was used to collect data. Interviewers took field notes during interview to gather verbal and non-verbal information together with voice recording to capture all information. As a standard procedure, a secure place was reserved for interview. Each participant meeting inclusion criteria was enrolled in the study. Data collection continued with the following available participant until the theoretical saturation was reached.

\section{Data analysis}


A verbatim transcription of interviews was performed, followed by their expansion with field notes and translation of Kinyarwanda transcripts in English by the researchers. The transcripts were read and reread until the researcher got familiar with the content. The translated text was analysed through gathering information around the key themes, thematic analysis, highlight the differences and similarities within codes and categories. Themes were coded whereby the key themes have been identified in a separate file. From the theme in a coding sort, the researcher interpreted and displayed data in a way that data from each code was detailed. From the details made from different themes in the created codes, the researcher was able to reduce data whereby only the essential concepts and interpretation were easy by explaining data's core meaning. Finally, a theme was identified, based on the underlying meaning throughout the codes, with three categories and seven sub-categories. To seek agreement and validation on findings, the analysed document has been shared within research group until consensus was achieved. Atlas ti software was used in the process of data analysis whereby codes were matched to the content from provided responses, an output produced with codes and quotation was used in analysis.

The study protocol was approved by the University of Rwanda College of Medicine and Health Sciences Institution Review Board (Ref: 363/CHMS/IRB/2019) before data collection.

Prior to conducting the interview, the participants were full educated in the study, potential benefits to them and the community; voluntary participation and withdrawal, the use of audio recorder, data management and confidentiality. There was no risk to participate. Participants consented verbally and a written consent was obtained. The written and recorded data was stored in a locked place and soft data was protected by the use of a password.

\section{Results}

\section{Characteristics of study participants}

Twenty-eight midwives were interviewed. All of them were aged 25-38 years (mean age 31.3 years) a half of them were married and a half were single. The majority had worked for more than three years $(n=$ 14), ten had worked between one and three years and six had worked for less than one year. The great majority of participants were registered A1 Midwives $(n=24)$ and four were A0 registered.

\section{Midwives knowledge on respectful maternity care during childbirth}

The RMC was launched in 2011 by White Ribbon Alliance and from this time it rooted in the international human right. The RMC community built a document, the human right of childbearing women (RMC Charter), with a common goal to demonstrate the respectful maternity care application as a fundamental human right in maternal health context. It has been used globally as a tool to help healthcare providers how maternity can be performed in accordance to the human right respect. It is in this purpose that it is 
used in this study to help researchers assessing perceptions and attitudes of midwives in providing RMC in selected settings.

\section{Midwives awareness on respectful maternity care}

In general, midwives working in maternity services from Masaka district hospital, Kacyiru district hospital and Muhima district hospital were knowledgeable on the RMC, they confirmed that they have had a course covering RMC during their curriculum, they have internal regulation and an orientation form guiding them about what information they need to share with their clients including their right to information, privacy and confidentiality.

"Yeah, we have learnt those rights in ethical course and we have internal regulations. We all know that clients need to be respected, informed about the procedure and confidentiality. For example, every client has her file; I am not allowed to share information from her file to others as it is her own information. Sharing it with others is prohibited". (Participant18)

\section{Knowledge on the seven rights of Respectful Maternity Care}

The majority of participants understood well seven rights which are the right to be free from harm and ill treatment, the right to information, informed consent and refusal, and respect for a woman's choices and preferences, including companionship during maternity care, the right to privacy and confidentiality, the right to be treated with dignity and respect, the right to equality, freedom from discrimination, and equitable care, the right to healthcare and to the highest attainable level of health, and the right to liberty, autonomy, self-determination, and freedom from coercion; stated in RMC. They make a self-presentation by introducing themselves to the clients and explain the basic information on the process of labour. Privacy was reported to be assured within existing infrastructure.

"I understand women's rights as the right to be provided with explanations/information to her health problem, being treated as a human being, playing a role in her healthcare, privacy; so that she can feel satisfied with the service provided."(Participant28)

In addition, participants perceived that they provide maternal healthcare services in equitable manner without any discrimination. For example, having health insurance or not does not affect the quality of service intended.

"From my experience, we provide healthcare for both insured and non-insured clients without counting on money as we need to save life and money comes later. This is the principal right but in some situations, clients may not receive a full range of healthcare services based on payment issue but I never observe it here, maybe in other hospital settings. We provide healthcare services and when client is unable to pay the social service intervenes."(Participant2)

\section{Knowledge on different kinds of mistreatment experienced by women during childbirth period}


Midwives had different views on existing kinds of women's mistreatment during childbirth period. They supported that mothers should be free from any harm during childbirth. The majority agreed that mothers need to be respected and they considered mistreatment as unprofessional practice.

"On my understanding, this should sound bad like beating a pregnant woman. She is in pain and this pain is observed even from her appearance. So I think we cannot cause harm to her, like shouting on her, even beating her. This is against the midwives' practices."(Participant2)

There are some shared experiences in abusive practices among midwives which can be classified in three forms. The first reported form of abusive practice is verbal by shouting on mothers, but midwives reported that they do not do it deliberately.

“(....) People do not do it from their willing of shouting on mothers. Meeting with uncooperative mother sometimes creates a bad reaction because if you do not shout on her, the outcome may be bad and you are not able to save the life of the baby, but this is a rare situation."(Participant25)

The second form of abusive practice is physical abuse by slapping on mothers particularly during second stage of labour and they considered it as a way to stimulate and help them pushing for a positive labour outcome.

"This small room is not comfortable for mothers and midwives. The second stage is a difficult moment for both mother and midwives. You all wish to have a safe baby. You may decide to let the mother doing whatever she wants but you may regret later; you try your best to have a safe baby. It is better to be asked why you slapped on a mother instead of responding to how the baby died. You do not slap on mother because you are against her, but for you need to have a safe baby".(Participant26)

The third form is psychological, from missing labour support. A narrative report from one midwife provided information on lack of support with mothers in labour based on their health status like having a contagious disease. Midwives in this case do not provide labour support as usual for fearing being in contact with mothers by prohibiting holding them in arms.

"A mother in labour needs support and you need to react for every step positively. You need to be on her side but sometimes, considering the existing number of contagious diseases, a mother may feel contraction and want to be hold in arms or other support like back massage; in this case, some midwives shout on them". (Participant16)

\section{Attitudes of midwives towards RMC during childbirth}

Midwives have different point of view on their attitude on RMC during childbirth. The majority report positive attitude towards RMC. They provide respectful maternity care services by respecting its underlying rights. Participants agree that really midwives support mothers during childbirth by not blaming them when they cry during contractions, but they try to educate them on how they can cope with contractions. 
"Yes of course". We provide respectful maternity care. Let us take an example; I cannot examine a mother in presence of others due to the respect on her privacy. Indeed, I need to provide all needed services in general. Sometimes there are mothers who cry when they are feeling contractions; in this case you do not blame them but we educate them on how she should cope with contractions" (Participant23)

In other hand, some participants report having observed some negative attitude among midwives while providing respectful maternity healthcare services and the most reported is shouting on mothers. Mothers are sometimes health cared in uncomfortable condition which is considered like unprofessional practice.

"Sometimes you can shout on the mother, or you do not provide the essential healthcare needed or performing suturing without anaesthesia and pain relief drugs and she may be in uncomfortable place, I think this also is not good for her". (Participant11)

\section{Challenges in providing Respectful Maternity Care}

The provision of respectful maternity care across three district hospitals presents some challenges: the software medical system, material, information, timely healthcare, privacy and infrastructure, client culture and religion, transfer process and other challenges.

\section{Medical system software and material}

some of the users of Community based health insurance $(\mathrm{CBHI})$ report that the process of check-up and registration into the system before receiving health care may cause a delay in intervention for some cases which need emergency and affects midwives who may be requested to advocate for mothers and go to explain the emergency case to the $\mathrm{CBHI}$ agent in order to provide the needed healthcare.

"This time, the system process requires for each insurer to be checked into system before receiving service as it is in conjunction with client identity card information. Here we always have a problem of internet; so that time, they cannot provide material or medicine to the client which delays the process as you are obliged to go yourself advocating for him/her while you have other many responsibility and this is time consuming".(Participant22)

In addition, the lack of labour monitoring material like CTG was reported in most of the participant and this result in late detection of foetal distress leads to increase in neonatal death rate.

"The main challenge in monitoring is the lack of cardiotocography (CTG). In our district hospital we do not have any. So the consequence you come and check on the foetal heart beat and you find baby dead inside from a mother who have been admitted with a live baby. This is what we call Intra-uterine foetal demise (IUFD)". (Participant21)

\section{Right to information}

Some participants think that clients receive information correctly. However, most of participants reported the shortage of midwives and high number of clients as a fact to lack of providing all needed information 
to women.

"The first challenge is that clients are too many considering to the number of midwives, beds and rooms. It means, when you have many clients, you provide short information, you do not enter in detailed and you cannot get another time to come back with a high number of activities. At this time, the client cannot get the chance for asking questions."(Participant28)

\section{Timely healthcare}

Most participants report that, due to overloaded work, they don't receive timely healthcare. The most activity affected is regular foetal monitoring.

"I cannot say that clients receive healthcare timely at 100\%. Depending on the high number of clients admitted. For example, hearing foetal heart rate among the mothers in room every 30minutes cannot be realized because you make a cycle and you reach on the last too late". (Participant6)

An existing strategy used for triage with different colour based on the severity of mother's condition is used whereby red symbolize an emergency case, the yellow colour symbolize the urgent case and green colour symbolize no-severe case but this can lead to a delay in health healthcare provision.

"Depending on this high number, the clients should come at hospital early morning and receive healthcare late. The one scored in green or yellow, you do not start with her as she is not considered as an emergency case, but progressively her issue becomes severe and falls in red colour while if she has been admitted timely her condition should not be severe as it is". (Participant6)

\section{Privacy and infrastructure}

Participants report that they are aware of the importance of privacy and try their best to respect privacy with existing infrastructure. However, privacy was reported to be affected by short space in maternity ward and gynaecological beds. The pregnant women are closed to each other and are obliged to share rooms and beds. This interferes with midwives' ability to provide private information to the mothers.

"There are many challenges. Let us talk about infrastructure and hospital capacity. When we receive many clients two mothers share the same bed which interferes with the privacy of the client, at that time the only thing to do as healthcare provider is to maximize the benefit from each one".(Participant18)

\section{Transfer process}

an overview on some cases that are more likely to be transferred from District hospital to Referral Hospital reported include peritonitis, post-partum haemorrhage, severe pre-term labour, having more than two previous uterine scar and severe pre-eclampsia. There are some requirements for transfer such as caution fee for those without the health insurance and availability of ambulance. 
Most participants report not having any challenges during external transfer process but there are few of them that revealed challenge like the lack of place in maternity ward which leads to refusal of transferred case with fact that there is no available place.

"The first, do you think that transfer the client is easy? Our challenge for transfer to referral hospital is another issue. There some cases which are refused and others are rejected from the criteria of admission like caution fee more likely to people without insurance which create a long discussion with delay."(Participant19)

Generally, the information gathered from our participants suggests that, the system of maternity health care service delivery is good, due to presence of key material and infrastructure; however, workload was persistently mentioned as major challenge.

\section{Discussion}

This study aimed to investigate the perceptions and attitudes of midwives towards the provision of respectful maternity care during childbirth in three district hospital, Kigali city. The main findings from this study reveal that midwives have knowledge on respectful maternity care from learnt course, internal regulations and international standards. However, some midwives reported some existing abusive practices such as shouting and slapping on mothers. Most of midwives assured their willingness to provide RMC in their workplace but most of them report having challenges which were mainly focused on their high workload with its impact on information sharing between midwives and mothers. In addition to that infrastructure factors affecting the privacy respect and sometimes transfer process may not be easy for them.

Midwives are knowledgeable on seven rights during childbirth. They admit the mothers after explaining them about their rights on information, privacy, liberty, and freedom as stated in the internal regulation. They have an orientation form guiding them whereby the mother is required to sign after being explained on the process of healthcare. A previous qualitative study conducted on exploring midwives' understanding of respectful and non-abusive maternal care in Ghana demonstrated similar information on how midwives provide information to mothers which include their right to healthcare, to information, to privacy, to consent, to best healthcare, to choice to treatment and the possible consequence to the chosen treatment [13].

Understanding respectful maternity care means that we consider every right among the seven rights stated in RMC charter. All participants have knowledge on right to freedom from harmful practices and they are committed to provide maternal healthcare free from harm as a mean of creating trustful environment between them and mothers which is supported by the findings from a qualitative systematic review and meta-synthesis on midwives perspectives on (dis)respectful intra-partum healthcare during facility-based delivery in sub-Saharan Africa whereby midwives are proud to be called midwives and are so called mamas because they have seen that providing respectful maternity care brings happiness for 
both midwives' and mothers and they feel more enthusiastic which encourage mothers to come to the facility [14].

The right to information was reported to be respected while providing RMC by most of the participants but few cases reported that there is challenge in providing full information to our clients due to overloaded work. These findings have some similarity with another qualitative study from mothers with complications in post-partum conducted in Rwanda "not taken seriously". This study revealed that women with complications during labour were not informed about their conditions before discharge and this support by information from one participant which states that she didn't know much about her sickness. She was just confused and think that she is going to die from her condition [15] and another participant from another qualitative phenomenological study on the meaning of a poor childbirth experience done in Rwanda, stated that the lack of explanations increased the feeling of being disrespectfully treated in her deep word stating that she was feeling like a cow they are taking to slaughter because midwives were looking at her without any explanation whereby she wonder if she is going to die and she thought that they are hiding important information from here [12].

The provided responses from our participants reveal that most of maternal healthcare services provided to mothers are based on privacy right but also few participants reveal that privacy is affected in many ways due to some challenges include sharing one bed for two mothers and having mothers in labour in general room. This is similar to what was reported from a study conducted in Tanzania on the prevalence of disrespect and abuse during facility-based childbirth, which reveal that lack of privacy during childbirth is $53 \%$ [6]. and a qualitative evidence synthesis on factors that influence the provision of intra-partum and postnatal healthcare by skilled birth attendants in low- and middle-income countries reveal some challenges in providing maternal healthcare respected privacy of the clients as stated by skilled birth attended that when they have heavy workloads, sometimes is difficult for them to provide privacy healthcare to all mothers [16].

The right to dignity and respect is reported to be respected by all participants but with some challenge like overloaded work. This has been reported even in the qualitative evidence synthesis on factors that influence the provision of intra-partum and postnatal healthcare by skilled birth attendants in low- and middle-income countries whereby heavy workloads report the limited time that health workers had for conducting thorough assessments of women, and only women suspected to have complications were examined [16]. In our findings, few participants reveal that it happens that a mother should be neglected by midwives who may not be responding to mothers call for help while discussing on their own issue. This is supported from another study conducted in Rwanda on the meaning of poor childbirth experience which reveal that some mothers are neglected by health healthcare providers by refusing being beside them during critical moments as explained by one participant that she was alone during labour, it happens for her to scream out to the nurse behind the curtain and the nurse didn't come for help. When she tried to call again to explain that she is feeling baby coming out, the nurse replied that she continue pushing till she gave birth alone [12]. 
The most predominant responses reveal that midwives provide timely healthcare but sometimes this is always affected by work overload as has been observed even from a qualitative evidence synthesis on factors that influence the provision of intra-partum and postnatal healthcare by skilled birth attendants in low- and middle-income countries, where by health workers' ability to provide timely, and to manage routine healthcare as well as emergency cases was jeopardised by heavy workload [16]. In additional to the work overload, from our finding, lack of some material like CTG machine for foetal monitoring and sometimes stock out on some medication affects the way midwives can provide timely healthcare.

During discharge process, all midwives report that mothers are not retained at hospital for payment issue because they all have social services for support from those mothers who do not have the way for hospital fee payment which is similar to the findings from a qualitative study on exploring midwives' understanding of respectful and non-abusive maternal healthcare in Ghana whereby midwives reported they have the social welfare in charge of discharged cases without payment as stated by the hospital policy [17]. But in other hand, from our findings we find that there are some existing challenges for those mothers who are not discharged recovered but now are transferred to the highest level (tertiary level); it happens that they may be refused by the referral level depending on bed availability or health healthcare providers miss communication.

Despite the reported knowledge on how respectful healthcare is provided, there are existing abusive practices revealed by a respected number of participants classified as physical or psychological.

For physical abusive practices, the most reported is slapping on mothers during pushing time and few reveal episiotomy suture without anaesthesia. This is also reported in East and Southern Africa from a direct observation of respectful maternity care study in five countries which reveal that mothers are slapped during labour specifically during the second stage of labour whereby midwives slapped on mother when it was difficult for them to deliver the placenta and mother was not tolerating removal of the placenta manoeuvre [8]. This kind of abusive practices is also support by the findings from a study conducted in Ethiopia on service providers' experiences of disrespectful and abusive behaviour towards women during facility based childbirth which reveal that among the provided responses a quarter of the respondents ever witnessing use of physical force or abrasive behaviour such as slapping or hitting labouring women [13].

In our findings, midwives report that hitting mothers help them to save their babies which are supported from what has been observed from a qualitative study on exploring midwives' understanding of respectful and non-abusive maternal healthcare in Ghana which reveal that mothers are hit for the purpose of saving not planned. Midwives reported that when the baby's head is crowned, they have to hit the childbearing woman because mothers tend to close their legs and midwives hit on them for the purpose to save baby's life. They report that doing this is not wrong for them as it was intended to save not to harm [17].

For psychological form of abuse, in our findings the most reported is shouting on mothers which is also supported by the findings from another study conducted in Rwanda on the meaning of poor childbirth 
experiences which reveal that midwives shout on mothers especially during pushing time as stated by one mother who said that the time she arrived at health facility, she meet a health healthcare provider and was in painful contractions. The midwife examining her shout on her the she started doubting on her help and she asked her mother and other companion support to leave that place which ended by giving birth on the floor [12].

Even though there are existing abusive practices and negative attitudes towards respectful maternal care, from our study findings the majority of midwives report that respectful maternity care is respected not $100 \%$ but they try their best to reach on the positive outcome of course by overcoming existing provided work place challenges and estimated at $80-85 \%$ reached.

These findings are comparable with the findings from a cross section study conducted in Rwanda on associations between perceptions of healthcare and women's childbirth experience where by $77.5 \%$ of the participants report a good experience on maternal healthcare which increase the rate of access to maternal healthcare [10] and findings from a study on exploring provider perspectives on respectful maternity care in Kenya: "Work with what you have" which provide an improved provider management, communication, and teamwork, resulting in better relations between providers and clients whereby healthcare providers reveal that communication among them was improved and goes beyond by reaching even on clients side which reduced some of their clients perceptions on disrespect and abuse [18].

\section{Methodological considerations}

The most strength of this study is that all midwives participated voluntary and consented for participation in the study. The sample can represent the population for the entire Kigali city. Data collection on the site was conducted by the trained persons who were not familiar with midwives thus they should respond openly. The study minimized the recall bias by asking the question related to midwives' daily practice. The participants were suggested to respond in their fluent language either Kinyarwanda or English. The records transcription was done by the team corrected data with the owner author shortly after data correction time so that every quotation was considered. All authors agreed on the content of the findings at each stage before moving to the next step.

\section{Conclusion}

This qualitative study describes the perceptions and attitudes of midwives towards the provision of respectful maternity care during childbirth in three district hospital, Kigali city. The findings show that midwives understand well the seven right of mothers as stated in the respectful maternal care charter. Some positive attitudes towards providing respectful maternal care such as providing information, privacy respect and providing all needed services, however there are also some negative attitudes like shouting on mothers and treating them in uncomfortable condition that were reported by participants. Participants reported some challenges associated with the provision of respectful maternity care which included high workload and lack of essential monitoring material like CTG. Therefore, there is a need to 
overcome the existing challenges in providing respectful maternity care by adjusting the midwives/client's ratio and providing essential material for labour monitoring in order to improve the quality of healthcare received by mothers during childbirth.

\section{Abbreviations}

RMC: Respectful maternity care

SDGs: Sustainable Development Goals

CMHS: College of Medicine and Health Sciences

IRB: Institutional Review Board

CTG: Cardiotocography

IUFD: Intra-uterine Foetal Demise

CBHI: Community-Based Health Insurance

USAID: United States Agency for International Development

\section{Declarations}

\section{Ethics approval and consent to participate}

This research protocol was approved by the University of Rwanda/ College of Medicine and Health Sciences Institutional Review Board (Ref: 363/CMHS/IRB/2019) before data collection.

To ensure confidentiality, the research team was trained on confidentiality and procedures to protect participant information. Anonymity of the interviewees' information was our main concern. All identifying information was stored in locked place. Audio recordings were used for the purpose of the transcript step and will be destroyed upon publication of the study.

A written informed consent was obtained from all participants in the study. The verbal explanation on the main objectives, procedures, risks, and benefits of the study was done to the interviewees. All participants have been provided an informed consent form that also detailed all process of the study including audio recording. The refusal of audio-recording was not considered as exclusion criteria to the study.

Participant who did not consent for audio-record participated and the investigator took notes instead of recording the interview. In total, two participants refused to be recorded.

\section{Consent for publication}

Not applicable 
Availability of data and materials

Full qualitative transcripts used and analysed during the current study are available from the corresponding author on request.

\section{Competing interests}

The authors declare that they have no competing interests.

\section{Funding}

No funding

\section{Authors' contribution}

All authors contributed to the drafting the manuscript. VU designed the study proposal and participated in all phases of the study. JPSS and AN assisted in data analysis and report writing while JH assisted in revising the manuscript. All authors read and approved the final manuscript.

\section{Acknowledgement}

Authors appreciate all midwives and nurses from three hospitals, data collectors, the Directors of the three District hospitals, and the University of Rwanda team for their support.

\section{References}

1. Balde MD, Bangoura A, Sall O, Balde H, Niakate AS, Vogel JP, et al. A qualitative study of women's and health providers' attitudes and acceptability of mistreatment during childbirth in health facilities in Guinea. Reprod Health. 2017;14(1):1-13.

2. Ishola F, Owolabi O, Filippi V. Disrespect and abuse of women during childbirth in Nigeria: a systematic review. PLoS One. 2017;12(3):e0174084.

3. Kruk ME, Leslie HH, Verguet S, Mbaruku GM, Adanu RMK, Langer A. Quality of basic maternal care functions in health facilities of five African countries: an analysis of national health system surveys. lancet Glob Heal. 2016;4(11):e845-e855.

4. Shakibazadeh E, Namadian M, Bohren MA, Vogel JP, Rashidian A, Nogueira Pileggi V, et al. Respectful care during childbirth in health facilities globally: a qualitative evidence synthesis. BJOG An Int J Obstet \\& Gynaecol. 2018;125(8):932-42.

5. Mannava P, Durrant K, Fisher J, Chersich M, Luchters S. Attitudes and behaviours of maternal health care providers in interactions with clients: a systematic review. Global Health. 2015;11(1):1-17.

6. Sando D, Ratcliffe H, McDonald K, Spiegelman D, Lyatuu G, Mwanyika-Sando M, et al. The prevalence of disrespect and abuse during facility-based childbirth in urban Tanzania. BMC Pregnancy Childbirth. 2016;16(1):1-10. 
7. Bell AF, Andersson E, Goding K, Vonderheid SC. The birth experience and maternal caregiving attitudes and behavior: A systematic review. Sex $\ \&$ Reprod Healthc Off J Swedish Assoc Midwives. 2018;16:67-77.

8. Rosen HE, Lynam PF, Carr C, Reis V, Ricca J, Bazant ES, et al. Direct observation of respectful maternity care in five countries: a cross-sectional study of health facilities in East and Southern Africa. BMC Pregnancy Childbirth. 2015;15(1):1-11.

9. Bohren MA, Vogel JP, Hunter EC, Lutsiv O, Makh SK, Souza JP, et al. The mistreatment of women during childbirth in health facilities globally: a mixed-methods systematic review. PLoS Med. 2015;12(6):e1001847.

10. Mukamurigo JU, Berg M, Ntaganira J, Nyirazinyoye L, Dencker A. Associations between perceptions of care and women's childbirth experience: a population-based cross-sectional study in Rwanda. BMC Pregnancy Childbirth. 2017;17(1):1-7.

11. WHO. Intrapartum care for a positive childbirth experience. World Health Organization. 2018;1-210.

12. Mukamurigo J, Dencker A, Ntaganira J, Berg M. The meaning of a poor childbirth experience-a qualitative phenomenological study with women in Rwanda. PLoS One. 2017;12(12):e0189371.

13. Asefa A, Bekele D, Morgan A, Kermode M. Service providers' experiences of disrespectful and abusive behavior towards women during facility based childbirth in Addis Ababa, Ethiopia. Reprod Health. 2018;15(1):1-8.

14. Bradley S, McCourt C, Rayment J, Parmar D. Midwives' perspectives on (dis) respectful intrapartum care during facility-based delivery in sub-Saharan Africa: a qualitative systematic review and metasynthesis. Reprod Health. 2019;16(1):1-16.

15. Semasaka JPS, Krantz G, Nzayirambaho M, Munyanshongore C, Edvardsson K, Mogren I. " Not taken seriously"-A qualitative interview study of postpartum Rwandan women who have experienced pregnancy-related complications. PLoS One. 2019;14(2):e0212001.

16. Munabi-Babigumira S, Glenton C, Lewin S, Fretheim A, Nabudere H. Factors that influence the provision of intrapartum and postnatal care by skilled birth attendants in low-and middle-income countries: a qualitative evidence synthesis. Cochrane Database Syst Rev. 2017;(11).

17. Millicent DV, Bemah BA, Emmanuel NK, Pascal A, Jody LR, Peter D. Exploring midwives' understanding of respectful and non-abusive maternal care in Kumasi, Ghana: Qualitative Inquiry. bioRxiv. 2019;708776.

18. Ndwiga C, Warren CE, Ritter J, Sripad P, Abuya T. Exploring provider perspectives on respectful maternity care in Kenya:"work with what you have." Reprod Health. 2017;14(1):1-13. 